\title{
Croissance et inflation en Argentine sous les mandatures Kirchner
}

\section{Crescimento e inflação na Argentina nos mandatos Kirchner}

Pierre Salama - Economista, Professor Emérito de várias universidade na França (site: http://perso. wanadoo.fr/pierre.salama). E-mail: pierresalama@gmail.com

\section{Resumé}

L'Argentine a renoué avec une industrialisation soutenue et une croissance élevée depuis 2003. Le taux d'investissement a augmenté, mais insuffisamment, les pressions sur les capacités de production installées demeurant fortes. Les dépenses sociales et les dépenses d'assistance ont cru sans que les grands équilibres macroéconomiques aient été affectés, à l'exception notable de l'accélération de la hausse des prix. La distribution des revenus est devenue moins inégale. La pauvreté a fortement baissé. La profondeur de la pauvreté ainsi que les inégalités parmi les pauvres ont également diminué selon la Cepal. Un bilan économique et social globalement bon avec toutefois des risques inflationnistes importants et des problèmes de gouvernance. La reprise économique extrêmement vive s'explique d'abord par l'abandon de la politique du taux de change réel apprécié des années quatrevingt-dix. Avec la très forte dévaluation du peso à la fin de 2001 et l'abandon consécutif du Plan de convertibilité établissant une parité nominale entre le peso et le dollar, l'Argentine plonge dans une crise très forte, mais dès 2003 le secteur industriel, protégé par le taux de change, connait une forte croissance. Cette reprise économique entraine l'augmentation de l'emploi et de la masse salariale malgré la réduction des salaires réels produite par la crise. Le rattrapage salarial ensuite et la poursuite de la hausse de l'emploi favorisent une augmentation de la consommation qui "tirent" la croissance. La hausse des profits et des perspectives de valorisation du capital conduisent à la hausse relative des investissements qui, jointe à l'augmentation des dépenses publiques alimentent la croissance comme nous essayons de démontrer dans ce texte.

\section{Mots-clés}

Développement. Croissance Economique. Industrialization. Argentine.

\section{Resumo}

A Argentina se recolocou com uma industrialização sustentada e um crescimento elevado desde 2003. A taxa de investimento aumentou, mas insuficientemente, as pressões sobre as capacidades de produção instaladas permanecem fortes. As despesas sociais e as despesas de assistência cresceram sem que os grandes equilibrios macroeconômicos fossem afetados, à exceção da notável aceleração da alta dos preços. A distribuição da renda tornou-se menos desigual. A pobreza diminuiu consideravelmente. $\mathrm{O}$ nível de pobreza, bem como as desigualdades entre pobres diminuíram igualmente, de acordo com a Cepal. Uma avaliação econômica e social no geral boa com, todavia, riscos inflacionistas importantes e problemas de governança. A retomada econômica extremamente viva se explica primeiro pelo abandono da política da taxa de câmbio real apreciada nos anos noventa. Com a forte desvalorização do peso no final de 2001 e o abandono consecutivo do Plano de convertibilidade que estabeleceu uma paridade nominal entre o peso e o dólar, a Argentina mergulha em uma crise muito séria, mas a partir de 2003, o setor industrial, protegido pela taxa de câmbio, vive um considerável crescimento. Esta retomada econômica leva ao aumento do emprego e da massa salarial, apesar da redução dos salários reais produzida pela crise. A equiparação salarial seguida da alta do emprego favorecem um aumento do consumo que "atraem" o crescimento. O aumento das vantagens e das perspectivas de valorização do capital levam ao aumento relativo dos investimentos que, com o aumento das despesas públicas alimentam o crescimento, como tentaremos demonstrar neste texto.

\section{Palavras-chave}

Desenvolvimento. Crescimento econômico. Industrialização. Argentina. 


\section{PRÉSENTATION}

L'Argentine a renoué avec une industrialisation soutenue et une croissance élevée depuis 2003. Le taux d'investissement a augmenté, mais insuffisamment, les pressions sur les capacités de production installées demeurant fortes. Les dépenses sociales et les dépenses d'assistance ont cru sans que les grands équilibres macroéconomiques aient été affectés, à l'exception notable de l'accélération de la hausse des prix. La distribution des revenus est devenue moins inégale. La pauvreté a fortement baissé. La profondeur de la pauvreté ainsi que les inégalités parmi les pauvres ont également diminué selon la Cepal. Un bilan économique et social globalement bon avec toutefois des risques inflationnistes importants et des problèmes de gouvernance.

La reprise économique extrêmement vive s'explique d'abord par l'abandon de la politique du taux de change réel apprécié des années quatre-vingt-dix. Avec la très forte dévaluation du peso à la fin de 2001 et l'abandon consécutif du Plan de convertibilité établissant une parité nominale entre le peso et le dollar, l'Argentine plonge dans une crise très forte, mais dès 2003 le secteur industriel, protégé par le taux de change, connait une forte croissance. La reprise économique entraîne l'augmentation de l'emploi et de la masse salariale malgré la réduction des salaires réels produite par la crise. Le rattrapage salarial ensuite et la poursuite de la hausse de l'emploi ${ }^{1}$ favorisent une augmentation de la consommation qui "tirent" la croissance. La hausse des profits et des perspectives de valorisation du capital conduisent à la hausse relative des investissements ${ }^{2}$ qui, jointe à l'augmentation des dépenses publiques alimentent la croissance. Un cercle vertueux se met en place d'autant plus facilement que la contrainte externe disparait grâce surtout au boom du prix des matières premières et à la hausse des exportations des produits industriels. La contrainte budgétaire est levée grâce aux rentrées fiscales provenant à la fois de la croissance et de l'alourdissement de la pression fiscale.

1 L'indice du niveau de l'emploi passe de 100 en 2001 à 94 en 2002, puis s'élève rapidement et se situe en 2007 à 118, et le salaire réel moyen est de 100 en 2001, il passe à 75 en 2002, 72 en 2003 pour retrouver quasiment son niveau de 2001 en 2007. Source : Ministère de l'économie de la Nation. Notons cependant que les créations d'emploi se font à un rythme moins soutenu dans les dernières années : sur les 3 millions d'emplois créés entre 2003 et 2010, 80\% l'ont été entre 2003 et 2007. L'augmentation de l'emploi est plus élevée dans le secteur public que dans le secteur privé : $4 \%$ en moyenne par an pour l'un contre 2,7\% pour l'autre. L'emploi public a augmenté en 2009 , année de crise alors que l'emploi privé baissait.

2 Rappelons qu'avec l'explosion du plan de convertibilité le système financier s'est effondré. Seuls l'autofinancement et le recours à des ressources "cachées " ont permis de financer l'essor de l'investissement. Sur les relations entre épargne et investissement en Argentine voir Marques Pereira J (2010, sous la direction de) : Les conditions de stabilité de la croissance, l'épargne et la distribution des revenus en Argentine, Rapport final pour la CDC, la CNP assurances et l'Université Pierre Mendes France. 
Tableau 1. Donnés statistiques de l'Argentine.

\begin{tabular}{|c|c|c|c|c|c|c|c|c|c|c|}
\hline & 2002 & 2003 & 2004 & 2005 & 2006 & 2007 & 2008 & 2009 & 2010 & $2011 *$ \\
\hline $\begin{array}{l}\text { Taux de } \\
\text { croissance du } \\
\text { PIB }\end{array}$ & -10.9 & 8.8 & 9 & 9.2 & 8.5 & 8.7 & 6.8 & 0.9 & 9 & $6^{*}$ \\
\hline Inflation** & 25.9 & 13.4 & 4.4 & 9.6 & 10.9 & 17.7 & 22.6 & 13.3 & 19.5 & $\mathrm{Nd}$ \\
\hline $\begin{array}{l}\text { Taux } \\
\text { d'investissement }\end{array}$ & 12.5 & 14.1 & 18.7 & 20.8 & 23.0 & 24.1 & 25.2 & 21.2 & 24.5 & 24.4 \\
\hline
\end{tabular}

Source : Annuaire statistique de la Cepal, 2010, pour 2011, prévisions (**) du FMI (Avril 2011) Global Economic Outlook. * prévision. **Les données pour l'inflation étant sous-estimées depuis 2007, celles présentées ici sont officieuses et sont estimées à partir des indices provinciaux.

Ce cercle vertueux est menacé cependant par une reprise inflationniste forte et risque de devenir un cercle vicieux. Dès 2007, la hausse du taux d'inflation est patente malgré les manipulations de l'indice des prix ${ }^{3}$. Ainsi la hausse du pouvoir d'achat des salariés est moins élevée que ce qui ressort des statistiques officielles, la réappréciation en terme réel du taux de change est supérieure à celle annoncée et la compétitivité des exportations manufacturières affaiblie. "Sauvé" par le soja, le modèle argentin est-il soutenable? Le risque inflationniste et la perte de compétitivité de l'industrie sont réels. Le modèle de développement, initié aux lendemains de l'échec du plan de convertibilité, rencontre là, des limites. Après les élections présidentielles d'octobre 2011, le nouveau gouvernement sera placé devant le l'alternative suivante: soit approfondir le modèle en maintenant une politique sociale plus "généreuse" et un taux de change déprécié, en espérant que la hausse de l'investissement, grâce à une politique industrielle plus audacieuse, réduira le taux d'inflation; soit, plus probablement, chercher à contenir le risque inflationniste en contrôlant davantage la demande globale, au risque de freiner la hausse des salaires et les dépenses sociales, et en laissant s'apprécier le taux de change. L'objet de cet article est de traiter de l'originalité de ce modèle, de ses succès et limites sur trois points essentiellement: la distribution des revenus, l'inflation et la compétitivité industrielle.

\footnotetext{
3 Face à l'emballement de la hausse des prix, les Autorités publiques ont modifié en 2006 la composition de l'indice des prix (Indec). Le nouvel indice sous-estime le taux d'inflation. La crédibilité de l'indice et des données divulguées par le gouvernement a baissé. La sousestimation de l'inflation conduit "mécaniquement" à une surestimation à la fois du taux de croissance calculé à prix constant et de la part des salaires dans la valeur ajoutée depuis 2007. Les indices calculés par les provinces n'ont pas été modifiés. Ils servent souvent de base pour évaluer la "vraie" hausse des prix. Il existe d'autres indices fournis par des universités, des sociétés privées. Les sociétés privées n’ont en général pas les moyens pour construire l'indice (enquêtes insuffisantes) et la construction de leur indice est en général opaque. Souvent opposées à la politique du gouvernement, elles ont tendance à surestimer le taux d'inflation. Plutôt que de faire une moyenne entre l'indice de l'Indec et celui de ces sociétés, nous préférons tenir compte de l'indice composé à partir des indices fournis par des provinces n'ayant pas modifié leur méthodologie.
} 
Des prévisions systématiquement erronées depuis la sortie du Plan de convertibilitéEffrayés par les mesures hétérodoxes décidées par le gouvernement argentin, des économistes argentins, dits de la City parce que liés aux institutions financières, pronostiquaient en 2002 le cours du dollar à 20 pesos, le retour de l'hyperinflation, la fermeture des banques. Une année plus tard, ce scénario catastrophe ne s'étant pas réalisé, ils expliquaient la forte croissance observée par un simple sursaut, une reprise "technique" suite à une profonde crise en 2002. Dès 2004, la croissance retrouvée devait fortement ralentir, selon ces Cassandres, à cause de l'insuffisance des ressources énergétiques, de la faiblesse des investissements et enfin de l'incertitude provoquée par les négociations sur la dette externe. 2005, 2006, 2007, 2008, pas une seule année sans des prévisions extrêmement pessimistes, tant au niveau de la croissance, du cours de change, de l'inflation. Plus récemment, en pleine crise internationale, ces mêmes économistes pronostiquaient de très graves difficultés pour l'Argentine et une incapacité de surmonter celles-ci. En octobre 2009, ils prévoyaient pour 2010 une croissance autour de 1,5\%, elle a été de 9\%, supérieure à celle du Brésil, le nouveau "bon élève " des institutions internationales... A prix constant, le PIB désaisonnalisé est passé de 237,3 milliards de pesos au dernier trimestre de 2002, année de creux, à 434,7 milliards de pesos au dernier trimestre de 2010 . Il a donc presque doublé en moins de dix ans. Les mesures hétérodoxes décidées sur le traitement de la dette publique allègent le fardeau des dépenses publiques au titre du service de cette dette mais ferment à l'Argentine l'accès aux marchés financiers internationaux. Le projet, avorté, d'établir un impôt progressif sur les exportations de matières premières, et la décision d'étatiser les fonds de pension, nourrissent la colère des économistes " de la City" et alimentent les fuites de capitaux ${ }^{4}$ Ces analyses pessimistes, voire catastrophiques, n’ont pas été l'apanage des seuls économistes argentins " de la City". On les retrouve chez nombre d'économistes liés aux institutions bancaires dans les pays industrialisés. Traitement hétérodoxe de la dette (externe et interne) considéré comme spoliateur, retour à l'Etat des fonds de pension, non respect de l'indépendance de la Banque centrale, conflits avec les propriétaires terriens, augmentation du poids des dépenses publiques, non respect du libre jeu des forces du Marché par des interventions jugées comme intempestives et désordonnées de l'Etat, autant de facteurs qui devaient conduire à des difficultés économiques croissantes et à des sorties de capitaux massives. Sur les sorties de capitaux, ces économistes ne se sont pas trompés, quant au reste, on ne peut qu'être surpris par leur cécité. Après avoir pronostiqué également une croissance faible, de graves difficultés économiques, les économistes de la BNP constatent aujourd'hui que leurs pronostics pessimistes ne se sont pas réalisés et notent que "la réalité a ...donné tort aux Cassandres ...le décalage entre les anticipations qui prévalaient alors et la situation ... observée aujourd'hui est si notable qu'il justifie un examen plus attentif". On ne peut s'empêcher de penser alors à l'humour du Nobel Niels Bohr qui, devant les difficultés de la prévision, écrivait: "prediction is very difficult, specially about the futur". Dans le cas de l'Argentine, on pourrait ajouter que l'aveuglement des économistes de la City s'explique pour l'essentiel par leur incapacité à envisager d'autres politiques efficaces que celles qu'ils préconisent ${ }^{6}$. L'ironie est que les profits des banques et les gains tirés de l'activité en Bourse ont été très élevés en $2010^{7}$. Cet aveuglement est partagé, en partie, par les économistes du gouvernement qui sous-estimant de moitié à la fois l'ampleur de la croissance et le taux d'inflation de 2010, ont eu la " bonne surprise " de constater des excédents de recettes fiscales...excédents qui, à la veille d'élection, peuvent servir de "cagnottes" pour financer des plans sociaux.

4 Sur cette question, voir l'étude de Gaggero J, Kupellan R. et Zelada M.A (2010). : La fuga de capitales II., Argentina en el escanario global (2002-2009). Documento de trabajo n²8 :1-66, CEFID*AR. De 2006 à 2009, la fuite des capitaux atteint 44 milliards de dollars, soit 93\% de l'ensemble des réserves accumulées par la Banque centrale durant cette période grâce aux excédents commerciaux. Elle atteint 7\% du PIB en 2008, alors qu'en 2007 et 2009, elle atteint 3,5\% et 4\% respectivement, et au début du mandat de N. Kirchner elle s'élève à un peu plus de 2\% en 2003, moins de $1 \%$ en 2004, légèrement négative en 2005 et un peu plus de $2 \%$ en 2006. (p.15 à 17).

5 Perracino-Guerin V (2011) : “Argentine, parti pour durer, finalement ?" BNP, revue EcoConjoncture $\mathrm{n}^{\circ} 2(1-21, \mathrm{p} .19)$. 


\section{LA DISTRIBUTION DES REVENUS DEVENUE MOINS INÉGALE DEPUIS 2003}

Il y a deux manières de mesurer la distribution des revenus ${ }^{8}$ : soit l'approche "fonctionnelle", soit l'approche "personnelle". La première analyse le partage du revenu entre profit et salaire ${ }^{9}$. Elle a donné lieu à de nombreux débats entre théoriciens classiques, marxistes, néoclassiques, keynésiens, cambridgiens et kaleckiens ${ }^{10}$. Elle est pour l'essentiel holiste. Nous nous limiterons ici à observer que la part des salaires dans la valeur ajoutée est très volatile au cours des quarante dernières années (voir Figure 1 ci-dessous). En 1976, date du coup d'Etat militaire et de la mise en place d'une politique économique très orthodoxe, la part des salaires est fortement réduite. Tout au long des années quatre-vingt, avec le retour de la démocratie, cette volatilité est la conséquence à la fois de la crise économique de longue durée, de l'hyperinflation, et de ses pauses en général brèves. Avec la venue

6 Ajoutons que ces prévisions catastrophiques sont souvent à l'origine de mouvements spéculatifs et ont pour objectif d'influencer les choix du gouvernement et l'orienter vers une libéralisation accrue des marchés.

7 Selon le quotidien d'opposition La Nacion du 31 decembre 2010: "El pais vivio un ano de fiesta financiera", la Bourse a été pour la seconde année consécutive parmi les cinq bourses les plus lucratives du monde et l'indice Merval a cru de plus de 50\%.

8 Il existe une littérature abondante sur ce sujet. On peut voir par exemple: Basualdo E. (2008): La distribucion del ingreso en la Argentina y sus condicionantes estructurales, Flacso - Clacso, Obervatorio latinoaméricano de geopolitica, p.4: 1-14; De la Garza E.et Neffa J.(éditeurs, 2010): Trabajo y modelos productivos en America Latina. Clacso, p.325 et suivantes; Lozano C, Rameri A., Raffo T. (2007): El debate de la distribucion, Instituto de estudios y formacion, CTA :1-30. On peut se référer également à Alfonsin J.M. et Tarallo Gariup E. (2008): Distribucion del ingreso en Argentina 1950-2007, Associacion del personal de los organismos de control (APOC) et à Cruzes G. et Gasparini L. (2009) Desigualidad en Argentina, una revision de la evidencia empirica, Desarrollo economico, $1^{\circ}$ partie Vol 48, $n^{\circ} 192: 395-437$ et $2^{\circ}$ partie Vol 49, $n^{\circ} 193: 3-29$ pour l'analyse de la distribution personnelle des revenus. Voir également Lo Vuolo (2010): Distribucion y crescimiento, una controversia persistente. Editeur Mino y Davila. Et notamment le chapitre 3, CENDA (2010): La anatomia del nuevo patron de crecimiento y la encrucijada actual, la economia argentina en el periodo 20022010, éditeur Cara o Ceca, voir notamment chapitres 4 et 6. Lindenboim J, Kennedy et Grana J (2010): “El debate sobre la distribucion functional del ingresso " in Desarrollo Economico, Vol 49, N¹96 (541-571).

9 Les entrepreneurs individuels, à savoir les travailleurs “ à leur propre compte ", pour la plupart “informels" en Argentine, disparaissent en tant que catégorie, leurs revenus, dits mixtes, sont convertis en salaires.

10 Pour expliquer les variations entre la part des salaires et celle des profits dans la valeur ajoutée, ces théories insistent, selon les auteurs, sur l'ampleur relative de l'investissement, l'importance de l'épargne, les différents types de progrès technique (biaisé ou non), l'ampleur des capacités de production oisives, la structure des coûts, l'importance des produits intermédiaires et surtout sur la recherche de taux de marge de la part des entrepreneurs et consécutivement sur le conflit distributif. Pour une présentation de ces différentes approches, voir Panigo D., Toledo F. et Agis E. (2008): Poder de mercado, crecimiento y distribucion functional del ingreso en Argentina, Document de Travail, Ministerio de economia y finanzas publicas: (1-59). 
au pouvoir du Président Menem au début des années 90, la hausse de la part des salaires s'explique par la fin durable de l'hyperinflation, puis la baisse qui a suivi par les politiques économiques libérales mises en œuvre. La crise économique initiée en 1998, l'effondrement du plan de convertibilité en décembre 2001 et la profonde crise de 2002, expliquent la chute vertigineuse de la part des salaires. Depuis 2003, cette part s'élève de nouveau, mais de manière relativement modeste ${ }^{11}$. On observe depuis cette date une amélioration de la part des salaires dans la valeur ajoutée et une distribution personnelle des revenus moins inégale.

Figure 1. Part des salaires dans la valeur ajoutée brute au prix de base, 1947-2006.

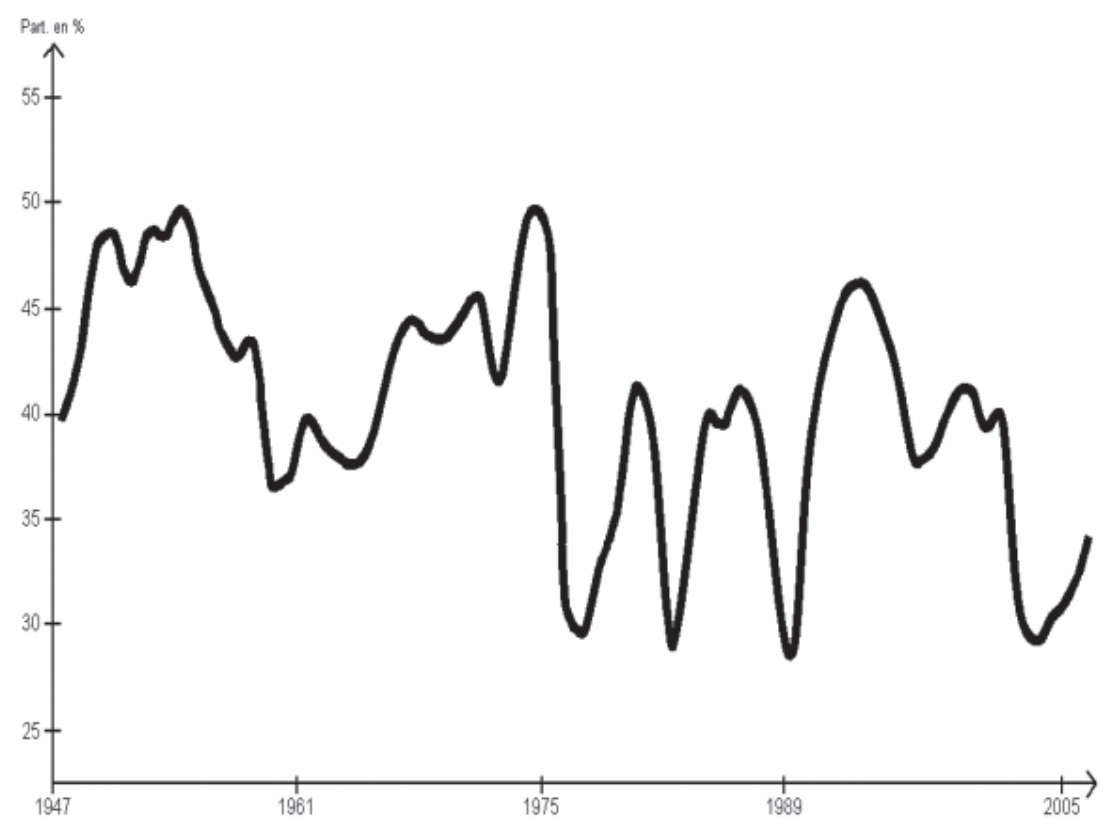

Source: CEPED, voir Lindenboim J et al. op. cit., p. 544.

L'analyse de la distribution personnelle des revenus s'est imposée avec la montée en puissance du paradigme de l'individualisme méthodologique. Elle est cependant riche d'enseignements car elle permet de comparer les parts respectives de revenus affectés à chaque décile, voir percile, de la population, surtout lorsqu'elle est complétée par une analyse de l'évolution des salaires réels et de la productivité du travail (voir Figure 2 ci-dessous). Au troisième trimestre de 2003, au moment où le président N. Kirchner accède au Pouvoir, les 10\% les plus riches de la po-

11 Sur les causes structurelles des changements de politiques économiques (expansionnistes versus libérales), liées aux spécificités des économies dites de la périphérie, voir les articles précurseurs de Diamand M, notamment celui de 1983 : "El pendulo argentino : hasta cuando ? " republié dans le blog de la revue Circus (http://grupolujan-circus.blogspot.com) 
pulation accaparaient 39,3\% du revenu national et les $10 \%$ les plus pauvres $0,7 \%$. Un an avant que ne s'achève la présidence de $\mathrm{C}$. Kirchner, au troisième trimestre de 2010, la part dans le revenu des 10\% les plus pauvres de la population double. Selon l'INDEC, le ratio des revenus des personnes tirés de l'emploi principal entre les $90 \%$ les plus riches et les $10 \%$ les plus pauvres est passé de 10 au $4^{\circ}$ trimestre 2003 à $8,3 \%$ au $4^{\circ}$ trimestre 2010, et le Gini entre les mêmes dates a baissé, passant de 0,471 à 0,390. Lorsqu'on prend l'ensemble des revenus, et non plus celui tiré de l'emploi principal, le premier ratio passe de 9,7 à 7,7 et le Gini de 0,525 à 0,439 (INDEC, 28 Mars 2011). La réduction des inégalités est donc indéniable, elle de l'ordre de 18\% sur la période et ceci malgré une courte phase d'augmentation des inégalités au second trimestre de 2010 liée à la crise de 2009 et aux effets de l'inflation. La réduction des inégalités est cependant moins élevée. Il ya en effet une sous-estimation officielle de la hausse du taux d'inflation à partir de mi-2007, or l'accélération de la hausse des prix affecte plus particulièrement le pouvoir d'achat des revenus des catégories les plus pauvres.

Bien que moins importantes aujourd'hui qu'en 2003, les inégalités demeurent extrêmement prononcées: les 10\% les plus pauvres gagnent 216 pesos par personne par mois en moyenne, soit au total 1,4\% du revenu national et les $10 \%$ les plus riches gagnent 7845 pesos, soit au total 32,9\% du revenu national, le double que ce que gagnent les $10 \%$ qui les précèdent.

\subsection{Des évolutions fortement contrastées du salaire réel et de la productivité du travail sur longue période}

Pour comprendre les évolutions récentes des salaires réels il faut les inscrire dans une longue période. Trois types d'analyse peuvent être faites: la première concerne l'évolution du salaire réel, la seconde celle de la productivité du travail et la troisième enfin le différentiel de taux de croissance entre ces deux variables.

Il y a une très forte volatilité du salaire réel sur une longue période. Depuis 1975, le salaire réel fluctue fortement autour d'un trend baissier comme on peut le voir sur le graphique ci-dessous. 
Figure 2. Evolutions du taux de salarisation, de salaire réel, du coût du travail et de la productivité moyenne sur longue période 1947-2006 (base 100: 1970).

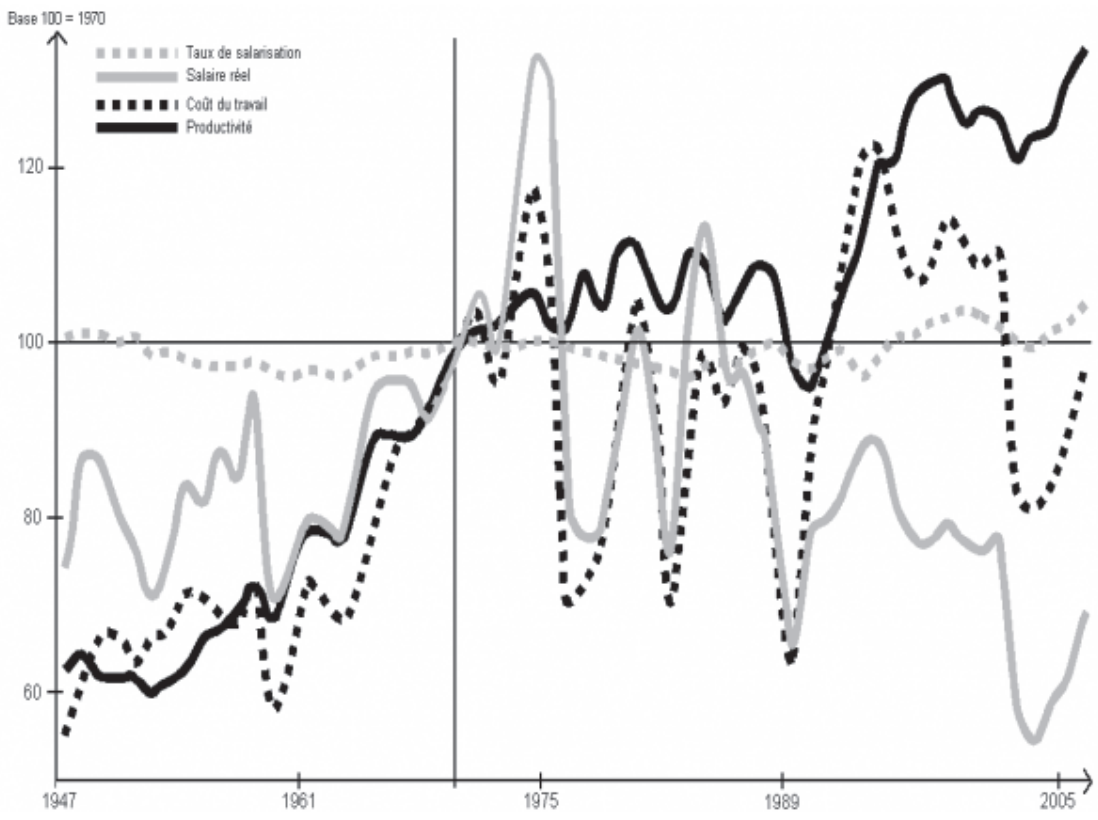

Source: élaboré par Lindenboim et alli à partir des données de l'INDEC, p.548.

De 1976 à 2003 la tendance suivie par le salaire réel est orientée à la baisse. La volatilité des salaires est très prononcée: l'indice du salaire réel passe de 132 en 1975 (pic) à 78 en 1977, après de nouvelles fluctuations, il atteint l'indice 110 en 1984, puis chute de nouveau et atteint un creux en 1989 avec un indice de 65. Depuis 2003, le salaire réel progresse et retrouve en 2006 le niveau atteint à la fin des années cinquante.

L'amplitude des fluctuations du salaire réel est considérable, alors que celle de la productivité est beaucoup plus faible ${ }^{12}$. La productivité du travail, après de nombreuses fluctuations, retrouve son niveau de 1976 en 1983 puis chute en 1988 pour retrouver en 1989 le niveau atteint en 1970. La croissance de la productivité étant plus soutenue dans la plupart des autres pays, l'Argentine perd de sa compétitivité industrielle. Avec la libéralisation des échanges décidée au début des années quatre-vingt-dix, cette perte de compétitivité se traduira par une désindustrialisation prononcée jusque 2003.

12 Sur ces questions traitées d'un point de vue théorique, voir Amico F., Fiorito A. et Hang G. (2011): "Producto potencialy demanda en el largo plazo: hechos estilizados y reflexiones sobre el caso argentino réciente", Documento de trabajo n³5, CEFID-AR, (1-90, voir p.21 à 28 sur Okun, Verdoorn et Kaldor). 
A partir de 1995 et jusque 2003, on observe un "décrochage" entre l'évolution du salaire et celle de la productivité. Ce "décrochage" s'explique essentiellement par les effets conjoints de la globalisation financière et commerciale et plus particulièrement par la manière dont celle-ci est menée. De 2003 à 2006, la hausse du salaire réel est un peu plus rapide que celle de la productivité du travail, soit approximativement $23 \%$ contre $10 \%$ sur la période. Le resserrement de l'écart entre les taux de croissance du salaire réel et de la productivité du travail s'explique à la fois par la reprise de l'activité industrielle et par les négociations salariales par branches, impulsées par le gouvernement, dans un contexte de contraintes externe et interne allégées par les excédents de la balance commerciale et du budget primaire.

\subsection{L'augmentation relative des emplois formels}

Selon les travaux de Arakaki et del Pilar Piqué ${ }^{13}$, portant sur 28 agglomérations urbaines, au second semestre 2003, 30\% des travailleurs étaient dans des entreprises dites micro (où dominent largement les emplois informels), $40 \%$ dans les entreprises petites et moyennes et 30\% dans de grandes entreprises (où à l'inverse dominent les emplois formels). Trois ans plus tard, la structure de l'emploi selon la dimension de l'entreprise a changé : soit respectivement 27\%, 40\% et 33\%. Les grandes entreprises emploient une part plus importante de travailleurs, or ce sont celles où les emplois informels sont les moins importants. Le poids des micro-entreprises, où dominent les emplois informels et en général peu qualifiés, régresse. Il y a donc en terme relatif une baisse des emplois informels. L'emploi total progresse de $43 \%$ sur la période, cette progression s'explique pour $23 \%$ par la hausse absolue d'emplois informels et pour 77\% par celle des emplois formels selon les auteurs.

La situation n'est pas la même selon les secteurs d'activité : dans l'industrie, la part relative des travailleurs informels est plus faible que dans les secteurs de la construction et du commerce, y compris dans les micro-entreprises. L'emploi total dans l'industrie progresse de 48\% et il s'explique pour $98 \%$ par la progression des emplois formels alors que la progression de l'emploi de plus de $120 \%$ dans la construction s'explique pour 35,5\% par la progression des emplois formels et celui de $40 \%$ dans le commerce par 75,5\% des emplois formels. L'évolution des disparités salariales n'est donc pas la même selon ces secteurs.

13 Arakaki G.A. et del Pilar Piqué (2009): "La disparidad salarial: una cuestion includible para discutir el modelo de crecimiento en la Argentina de la post-convertibildad”, miméo (CEPEDIIE-UBA). 
Entre 2003 et 2006, le salaire mensuel moyen progresse de 904 pesos pour les travailleurs enregistrés (formels) à 1372 pesos (soit une augmentation de $52 \%$ ) et pour les travailleurs non enregistrés (informels) de 493 pesos à 755 pesos (soit une augmentation de 53\%) (voir Tableau 2). L'éventail des salaires entre les emplois formels et informels tend à se maintenir sur la période.

Les salariés des grandes entreprises, à qualification équivalente, gagnent en général davantage que les salariés des petites et moyennes entreprises et ces derniers davantage que ceux employés dans des micros entreprises. Les salariés ayant des emplois informels dans les grandes entreprises gagnent davantage que les salariés ayant des emplois informels dans les micro-entreprises, mais la progression de leurs revenus est plus faible sur la période considérée (26\% contre 56\% dans les micro-entreprises).

Au total, la disparité salariale entre les emplois formels et informels tend à se maintenir sur la période considérée et la progression forte des salaires en moyenne s'explique pour partie par la transformation de la nature des emplois vers davantage de formalité. A la lumière de données moins complètes que celles utilisées pour la période 2003 - 2006, on peut penser que ces caractéristiques se maintiennent dans les années 2007 à 2010, malgré la crise de 2009.

Tableau 2. Evolution du salaire réel selon la taille des entreprises et la nature des emplois, 2003-2006.

\begin{tabular}{l|r|r|r|r}
\hline & $2^{\circ}$ trim. 2003 & $2^{\circ}$ trim. 2004 & $2^{\circ}$ trim. 2005 & $2^{\circ}$ trim. 2006 \\
\hline Total & 736.5 & 782.6 & 939.5 & 1154.1 \\
\hline Formels & 904 & 942.6 & 1123.3 & 1372.3 \\
\hline Informels & 492.6 & 527.2 & 617.7 & 754.8 \\
\hline Micro-entreprises & 476 & 522.2 & 643.8 & 721.1 \\
\hline Formels & 646.9 & 716.1 & 911 & 1038.7 \\
\hline Informels & 399.5 & 433.1 & 510 & 624.7 \\
\hline Petites - moyennes & 683.5 & 741 & 877 & 1073 \\
entreprises & & & & \\
\hline Formels & 781.3 & 837.7 & 986.5 & 1189.3 \\
\hline Informels & 532.4 & 571.9 & 681.2 & 857.9 \\
\hline Grandes entreprises & 1958.2 & 1078.2 & 1264.7 & 1568.8 \\
\hline Formels & 1101.2 & 1114.1 & 1311.8 & 1633.1 \\
\hline Informels & 802.8 & 838.2 & 901.3 & 1008 \\
\hline
\end{tabular}

Source: Arakaki et alii, op.cit.p.10, selon les données de l'EPH. 


\section{LE CERCLE VERTUEUX DE LA CROISSANCE MENACÉ PAR LE RETOUR DE L'INFLATION?}

\subsection{La croissance devenue vertueuse}

Le contexte international a changé avec la forte croissance de la Chine et de l'Inde. La hausse du cours des matières premières, provoquée par la demande de ces pays, assouplit durablement la contrainte externe et interne de l'Argentine, grâce aux excédents de la balance commerciale et aux rentrées fiscales liées à l'exportation de matières premières. Le cercle vicieux dans lequel se trouvaient de nombreux pays comme l'Argentine tend alors à devenir vertueux.

1) Le cercle vicieux se manifeste par une forte volatilité des principales variables. Les variables-clé expliquant ces enchaînements sont les variations du taux de change et le faible niveau de la productivité du secteur industriel. Une croissance à taux élevé provoque un déficit de la balance commerciale et une dévaluation à terme de la monnaie nationale. D’une manière générale, la dévaluation diminue les salaires exprimés en devise. Dans les économies semi-industrialisées, son impact sur les exportations est modéré pour deux raisons: l'élasticité de la demande étrangère de produits primaires ne dépend pas des dévaluations puisque la cotation de ces produits se fait en devises; avec un niveau de productivité du secteur industriel faible, les capacités de réponse de ce dernier à une baisse des prix en devise sont réduites. La dévaluation a donc un effet surtout sur les importations. Les importations, devenues plus coûteuses, provoquent une hausse des prix qui, diminuant les salaires réels exprimés en monnaie locale, aiguise le conflit distributif. Pour contenir cette hausse des prix, la réduction des dépenses publiques, la limitation des salaires nominaux en deçà du taux d'inflation et la hausse des taux d'intérêt sont très souvent recommandés ${ }^{14}$. L'application de ces mesures précipite la récession. Celle-ci est à l'origine de politiques expansionnistes conduisant à de nouvelles dévaluations. Le profil de la croissance est alors de type "stop and go". La croissance s'inscrit dans un cercle vicieux comme l'ont souligné les travaux de Diamand (op.cit.) ainsi que de nombreuses études de la Cepal.

2) Le cercle vicieux tend à devenir vertueux. Depuis 2003, et à l'exception de l'année 2009, le taux de croissance est élevé et régulier et s'accompagne d'excédents de la balance commerciale. L'analyse présentée ci-dessus ne peut plus s’appliquer et

14 Contenir la demande globale pour limiter la hausse des prix n'est pas l'apanage des seuls économistes appartenant au mainstream. Des économistes, comme Frenkel et Damill en Argentine (op.cit. p.16 et 17), considérés comme keynésiens, légitiment une politique visant à contenir la demande globale par un ralentissement des dépenses publiques, afin de contrebalancer les effets inflationnistes d'une politique de change relativement déprécié. Voir Frenkel R. et Damill M.: Las politicas macroeconomicas en la evolucion reciente de la economia argentina, Nuevos documentos CEDES, N65 (1-89), disponible sur le site du CEDES. 
ceci pour trois raisons : 1 / la hausse des termes de l'échange des matières premières est durable et l'Argentine "profite" de la hausse du cours du soja, suscitée par la demande croissante des pays asiatiques, en concentrant l'essentiel de ses terres à la production de ce produit de rente; 2 / quelques branches de l'industrie ont atteint des niveaux de productivité qui se rapprochent de ceux des pays développés et sont davantage capables de profiter d'un taux de change déprécié pour exporter davantage; enfin 3/ la politique suivie par le gouvernement s'apparente à une politique de changes multiples. Elle vise à maintenir un taux de change déprécié. Celui-ci profite à l'industrie en lui procurant un protectionnisme par les changes en rendant plus difficile les importations, et en facilitant ses exportations. Les taxes ("rétentions") calculées sur le prix international des exportations de matières premières réduisent les revenus en monnaie locale des exportateurs. Leurs effets s'apparentent à ceux d'une appréciation du taux de change pour ces exportateurs. L'inflation importée induite par le maintien de la dépréciation de la monnaie et par la hausse du cours des matières premières, dont l'énergie et les produits alimentaires, peut être contenue grâce à des subventions financées par les ressources provenant des "rétentions".

Le cercle vicieux devient alors vertueux grâce aux modifications du contexte international et national. Forte croissance, amélioration de l'emploi et des salaires, et au lieu des déficits jumeaux, externe et interne, excédents de la balance commerciale et surplus primaire du budget (hors dépenses au titre du service de la dette). C'est ce cercle vertueux qui fonctionne depuis la fin du plan de convertibilité. La reprise récente de l'inflation, depuis 2007, menace cependant de le transformer en cercle vicieux dans les années à venir. Elle ne s'explique pas seulement par le maintien d'un taux de change déprécié. L'hétérogénéité du tissu industriel, en dépit d'une certaine modernisation depuis 2003, les formes nouvelles du conflit distributif salaire-profit, les salariés augmentant leur pouvoir de négociation avec la poursuite de la croissance ${ }^{15}$, expliquent également l'accélération de la hausse des prix.

\subsection{Un retour de l'inflation préoccupant}

La littérature est abondante sur l'inflation et ses causes. Les courants néoclassiques et monétaristes insistent sur l'excès de demande par rapport à l'offre, excès dus à des dépenses publiques trop importantes, à des revenus du travail trop conséquents, excès qu'il conviendrait de contenir. Les approches keynésiennes et marxistes insistent sur les conditions de l'offre et sur le conflit

15 Sur cette question, voir Amico F. et Fiorito A. (2010): "Exchange rate policy, distributive conflict and structural heterogeneity. The Argentinean and Brazilian cases", Actes du Colloque Sraffa, (1-26, p.13), dans http://grupolujan-circus.blogspot.com 
distributif. Ce sont ces dernières que nous retiendrons ici. Selon ces approches, la société est hiérarchisée : les entrepreneurs agissent sur deux marchés, celui des biens où ils fixent les prix, et celui du marché du travail où ils achètent le force de travail. Les salariés agissent, eux, sur le seul marché du travail. Dans un marché monopolistique, les prix sont déterminés par les entreprises. L'offre est donc première (plus précisément l'investissement), la demande seconde. Le marché peut ensuite corriger ces prix si la demande est insuffisante par rapport à l'offre et inversement. La fixation des prix reflète ainsi la stratégie des entreprises: elle dépend de leur pouvoir de monopole et a pour objectif de renforcer ce pouvoir dans un horizon temporel donné, via l'investissement permis par le taux de marge recherché. Dans cette approche kaleckienne: le taux d'inflation exprime la divergence entre la nécessité d'obtenir un certain taux d'investissement correspondant au montant d'investissement désiré par les entrepreneurs et le taux de salaire réel qu'ils doivent payer aux salariés. Le taux d'inflation exprime également la divergence entre le salaire réel existant et celui désiré par les salariés. Selon Ramos "Le rôle de l'inflation est de rendre compatible ex-post la distribution des revenus qui, ex-ante, ne permettait pas l'équilibre sur le marché des biens".

Des chocs externes peuvent agir sur le taux d'inflation et relancer, voire entretenir le conflit distributif. La hausse du cours des matières premières est un facteur inflationniste, celle des produits agricoles agit plus fortement sur le pouvoir d'achat des catégories modestes dans la mesure où leur panier de consommation comprend une part importante de produits de subsistance, celle des produits énergétiques se diffuse dans le tissu industriel et dans les transports sauf à les subventionner très fortement. Ce fut le cas dans les années 2000. Un taux de change maintenu déprécié constitue un canal particulièrement favorable à la diffusion de l'inflation importée. Instrument pouvant servir à protéger l'industrie, c'est-à-dire à favoriser la compétitivité et la rentabilité de pans entiers de celle-ci, le taux de change déprécié permet à la hausse du cours des matières premières de se déployer sur le marché interne et d'amputer le pouvoir d'achat des salariés ${ }^{16}$, relançant ainsi le conflit distributif et nourrissant ainsi l'inflation lorsque les salariés parviennent à compenser la perte de leur pouvoir d'achat.

Plus concrétement, et à la lumière de ce qui vient d'être énoncé, on observe que jusque Mai 2008 l'indice des prix et l'indice des prix des produits

16 Très souvent les producteurs fournissent le marché interne et le marché externe de des produits alimentaires. Ils sont dès lors tentés de pratiquer les même prix sur le marché interne que ceux qu'ils obtiennent sur le marché externe. 
"échangeables" ${ }^{17}$ évoluent parallèlement (voir Figure 3). Une part de la hausse des prix est attribuable à l'inflation importée et cette part semble être constante jusque Mai $2008^{18}$. L'écart entre les évolutions des deux indices s'accentue jusque janvier 2009, diminue fortement puis reprend de l'ampleur à partir de septembre 2009. La divergence entre les deux indices est préoccupante. La politique de change ne peut expliquer totalement l'accélération de la hausse des prix et ce d'autant plus que la taux de change réel commence à s’apprécier depuis 2010. L’appréciation du change réel devrait être déflationniste or l'inflation croit et ceci malgré les fortes subventions accordées aux tansports et à l'énergie. Entre janvier 2009 et janvier 2010, l'inflation en pesos est de 12\% alors même que les prix des produits exprimés en dollars sont relativement stables. L'impression qui se dégage est que si l'inflation se déploie c'est parce que le tissu industriel reste encore trop hétérogène et en partie archaique malgré la hausse du taux d'investissement et celle de la productivité du travail. Face à une demande croissante, l'offre ne peut répondre suffisamment vite. C'est donc davantage du côté d'un approfondissement de la modernisation du tissu industriel, et donc de l'offre, que du côté de la demande qu'il faut chercher les remèdes à une inflation devenue trop élevée.

C'est l'insuffisante modernisation de l'ensemble de l'appareil industriel argentin et surtout l'absence de nombreux segments des lignes de production absents, détruits par la désindustrialisation des années quatre-vingt et quatre-vingtdix qui expliquent que malgré une compétitivité moyenne améliorée, le déficit de la balance commerciale des produits industriels persiste. C'est ce que nous allons analyser.

17 L'indice du niveau génral des prix utilisé ici n'est pas celui de l'INDEC pour les raisons indiquées précédemment. C’est un indice synthétique conçu à partir des indices des différentes provinces, qui eux n’ont pas été "reconstruits". L'indice des produits "échangeables" est un indice établi à partir des prix internationaux (moyenne entre les prix des exportations et des importations argentins) évalués au taux de change en vigueur. Il constitue un bon indicateur de l'inflation importée.

18 L'indice des prix des produits "échangeables" ne se répercute pas intégralement sur l’indice général des prix pour deux raisons, l'une parce que les importations sont pour une part importante des produits intermédiaires ou des biens d'équipement servant à produire des biens, l'autre parce que le gouvernment essaie d'atténuer l'effet de la hausse spectaculaire de prix des produits alimentaires et énérgétiques par des subventions sur ces produits. En 2008, sur 31 milliards de pesos de subventions, 16,2 milliards étaient destinées au secteur énergétique, 8,7 au secteur des transports et 3,8 au secteur alimentaire. (source ASAP in Frenkel R. et Damill M.(2009), op.cit., p.64, voir aussi Salama P (2008): "Argentine: la hausse des matières premières agricoles, une aubaine?”, publié en espagnol dans la revue Comercio exterior (2008), disponible sur ma page. 
Figure 3. Evolutions des indices de prix.

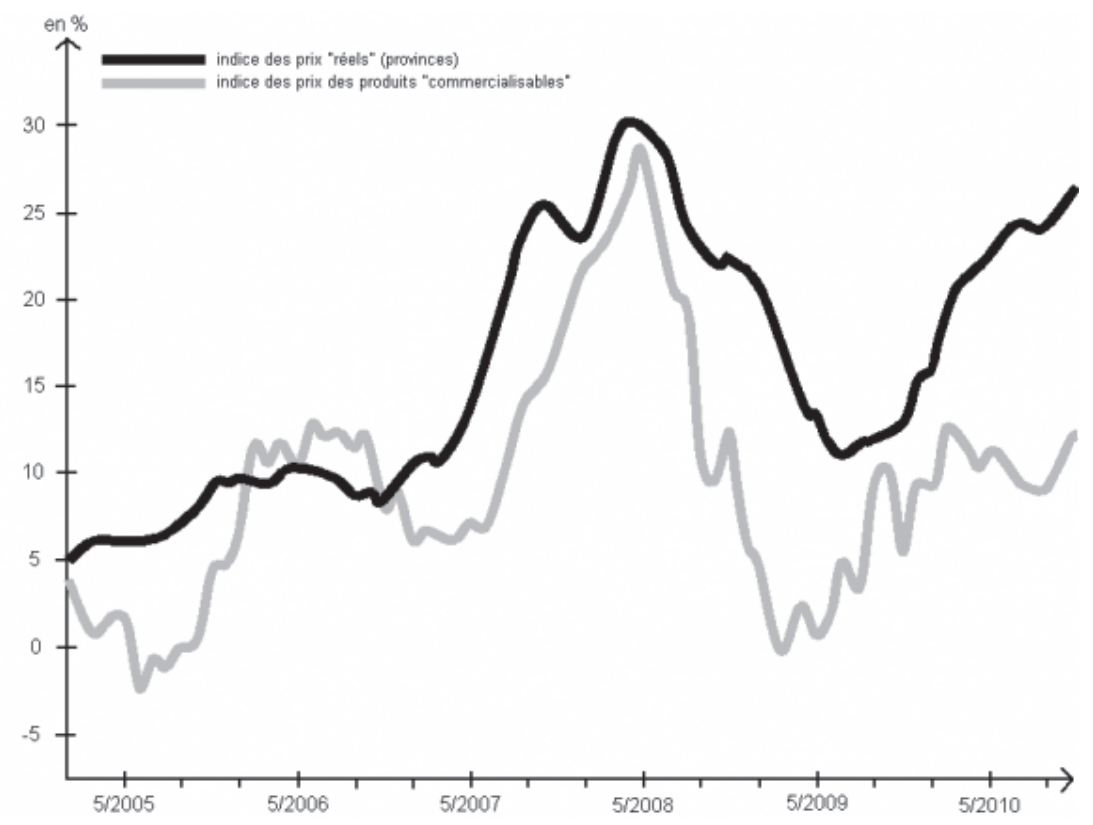

Source: graphique construit par le groupe "Inflacionheterodoxa" (2011): http:/ inflacion-heterodoxa. blogspot.com/

\subsection{Compétitivité et balance commerciale de l'industrie}

Malgré la modernisation sensible de quelques branches du secteur industriel, celui-ci reste profondément hétérogène en termes de productivité. C’est ce qui explique en partie que malgré l'amélioration de la productivité moyenne et le taux de change favorable, les échanges de produits industriels avec le Brésil puissent être déficitaires.

1) On mesure généralement la compétitivité d'un pays, hors secteur de rente, par le coût unitaire du travail, c'est-à-dire par le rapport salaire/productivité. Il suffit que le différentiel de salaires entre le pays exportateur et le pays importateur soit inférieur au différentiel de productivité pour que la compétitivité - prix soit favorable pour un taux de change donné. Une dépréciation du taux de change améliore mécaniquement la compétitivité-prix pour un coût unitaire du travail donné. Par ailleurs, la dépréciation de la monnaie nationale, en rendant plus coûteuses les importations, protège des pans entiers de l'industrie, favorise une intégration plus importante des lignes de production et accroit la valeur ajoutée de celles-ci. En ce sens, elle facilite une densification du tissu industriel. 
2) Malgré l'évolution favorable du coût unitaire du travail, la forte dévaluation et le maintien d'un taux de change déprécié, la balance commerciale des produits industriels de l'Argentine est négative, y compris avec le Brésil.

Avec la sortie du plan de convertibilité fin 2001, le coût horaire argentin du travail dans l'industrie, exprimé en dollars, a baissé fortement. Selon Abramovitch et $\mathrm{al}^{19}$, le coût horaire du travail exprimé en dollars en Argentine était équivalent à 31\% de celui aux Etats-Unis en 2001. Après la dévaluation, il ne correspond plus qu'à 11\% en 2002. Ce n'est qu'en 2008 qu'il retrouve le niveau d'avant la dévaluation, soit $31 \%{ }^{20}$. Les mêmes tendances se dégagent de la comparaison avec le Brésil (voir le tableau 3 ci-dessous).

Tableau 3. Coûts unitaires du travail comparés en Argentine, aux Etats-Unis et au Brésil.

\begin{tabular}{l|c|c|c|c|c|c|c}
\hline & \multicolumn{2}{|c|}{2001} & \multicolumn{2}{c|}{2002} & \multicolumn{2}{c|}{2008} & $\mathbf{2 0 0 1 - 0 8}$ \\
\hline Pays & $\begin{array}{c}\text { u\$s } \\
\text { courant }\end{array}$ & $\begin{array}{c}\text { Índice } \\
\text { relatif } \\
(1)\end{array}$ & $\begin{array}{c}\mathrm{u} \$ \mathrm{~s} \\
\text { courants }\end{array}$ & $\begin{array}{c}\text { Índice } \\
\text { relatif } \\
(1)\end{array}$ & $\begin{array}{c}\mathrm{u} \$ \mathrm{~s} \\
\text { courants }\end{array}$ & $\begin{array}{c}\text { Índice } \\
\text { relatif } \\
(1)\end{array}$ & $\begin{array}{c}\text { Variatión } \\
\text { Índice relatif }\end{array}$ \\
\hline Argentine & 8,15 & 1,00 & 2,98 & 1,00 & 9,89 & 1,00 & $0 \%$ \\
\hline Etats-Unis & 25,9 & 0,31 & 27,01 & 0,11 & 32,26 & 0,31 & $-3 \%$ \\
\hline Brésil & 3,6 & 2,26 & 3,07 & 0,97 & 8,28 & 1,19 & $-47 \%$ \\
\hline
\end{tabular}

(1) l'indice relatif est le quotient entre le coût horaire du travail argentin et celui d'un autre pays Source: Abramovitch et al (p. 5).

Les trois graphiques suivants montrent que le coût unitaire du travail en dollars a baissé fortement en 2002 en Argentine, davantage qu'au Brésil et qu’il remonte ensuite moins en Argentine qu'au Brésil. On observe que l'indice de taux de change réel pondéré par le coût unitaire du travail entre ces deux pays est relativement stable entre 2003 et 2008. Cette stabilité relative, comme le font remarquer Abramovitch et al (p.10), n’est pas due à la stagnation des salaires en Argentine puisque ceux-ci croissent de 38,7\% entre 2001 et 2010. Le différentiel de croissance des salaires entre les deux pays est donc compensé par celui de la productivité et par l'appréciation du real par rapport au peso (p.10).

19 Abramovich A.L., Lavopa A. et Serino L.A. (2011, 1-23): “ Dynamica salarial y tipo de cambio real, un analisis exploratorio de los costos laborales relativos de la industria argentina y sus principales socios comerciales en el periodo 2001-2010", mimeo, AEDA, $2^{\circ}$ Congrés annuel: Lineamentos para un cambio estructural de la economia argentina. Desafios del bicentenario. Sauf indications contraires, toutes les données qui suivent sont tirées de cette étude.

20 Deux facteurs expliquent cette remontée: une légère réappréciation du peso face au dollar, une augmentation des salaires réels supérieure à celle observée aux Etats-Unis. 
Figure 4. Evolution du coût unitaire du travail (CLU) évalué en dollars courants.

Argentine

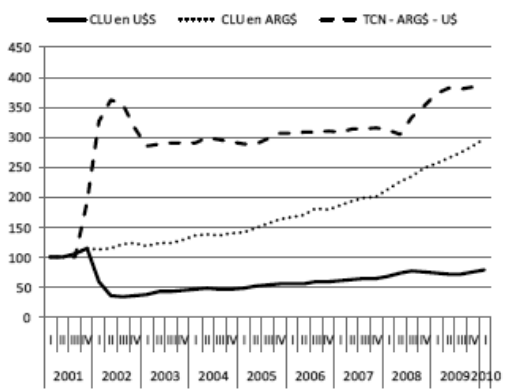

Brésil

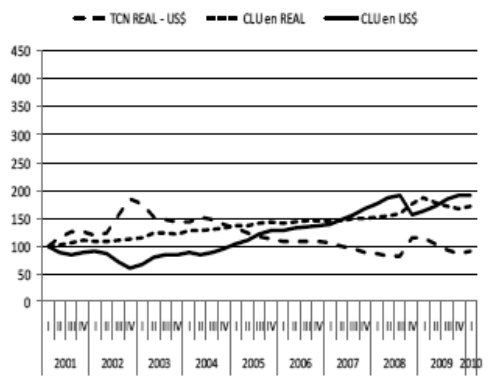

Source: Abramovitch et al., p.10, élaboration des auteurs à partir des données officielles.

Figure 5. Indice du taux de change réel bilatéral Argentine - Brésil (TCR base 100 en 2001) déflaté par les coûts unitaires du travail (CLU) respectifs.

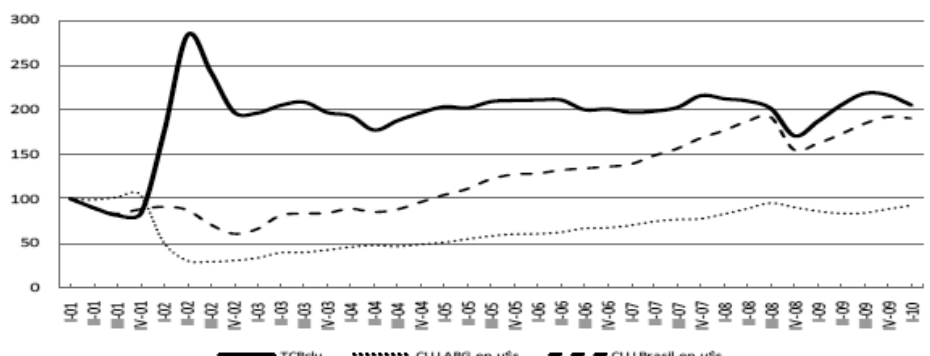

Source: Abramovitch et al., p.10, élaboration des auteurs à partir des données officielles.

Apparemment, la situation ne s'est pas détériorée du point de vue de la compétitivité. Pourtant, dès 2003, le solde commercial entre les deux pays s'inverse. De 1994 à 2002, les échanges commerciaux entre l'Argentine et le Brésil sont excédentaires pour l'Argentine, mais de 2003 à 2011, ils sont devenus déficitaires ${ }^{21}$. C'est un paradoxe. L'évolution du coût unitaire du travail pondéré par le taux de change ne devrait pas conduire à ce résultat.

3) Cette évolution s'explique principalement ${ }^{22}$ par la très forte désindustrialisation qu'a connue l'Argentine de 1976 à 2003. Le tissu industriel

21 Voir IEDI (2011): Argentina, Brasil e o Mercosul.

22 Dans le cadre de cet article nous ne pouvons traiter des comportements différents des industriels par rapport aux taux de marge désirés. Il est probable que le déficit commercial s'explique aussi en partie par les conséquences d'un accès plus rare au crédit en Argentine qu'au Brésil. Le taux de marge recherché alors pour financer les investissements pourrait être plus élevé en Argentine qu'au Brésil et il en résulterait une hausse relative des prix qui aurait un impact négatif sur la compétitivité. De même le contexte plus inflationniste en Argentine qu'au Brésil devrait aussi nourrir des anticipations inflationnistes minant la compétitivité-prix. 
argentin est moins dense que celui du Brésil. Malgré une réindustrialisation et une modernisation depuis 2003 en Argentine et une tendance à la désindustrialisation au Brésil, l'appareil industriel argentin n'est pas en mesure de résister suffisamment ${ }^{23}$. Dans de nombreux secteurs, l'élasticité de l'offre par rapport aux prix est rigide d'où des difficultés à exporter. L'élasticité de sa demande par rapport aux prix est également rigide d'où une propension à importer malgré des hausses de prix. On a pu ainsi évaluer, sur la période 1994 -2009, que pour 1\% de croissance du PIB argentin, les importations en provenance du Brésil varient de 4\%

L'hétérogénéité de l'appareil industriel est source d'inflation par les coûts. C'est donc sur le tissu industriel que les efforts doivent être portés en jouant à la fois sur la modernisation et sur la valeur ajoutée. Même si la situation externe de l'Argentine n'est pas inquiétante, elle est préoccupante. Le solde négatif de la balance commerciale des produits industriels, et plus particulièrement avec le Brésil, constitue un signal important de la fragilité de l'Argentine.

\section{CONCLUSION}

Le taux de croissance du PIB est très élevé, le pouvoir d'achat des salariés est en hausse, même lorsqu'il est corrigé par un indice des prix plus fiable, l'emploi

23 Le déficit de la balance commerciale des produits industriels ne doit pas cacher une forte croissance des exportations de ces produits. Si on prend pour base 100 l'année 1993, les exportations de produits manufacturés d'origine industrielle (MOI) atteignent au quatrième trimestre de 2010, 733,6 en valeur et 587,9 en volume, celles d'origine agricole 517,7 et 297,5 respectivement et les exportations de produits primaires, hors combustible et énergie, 313,1 et 176,5 respectivement (INDEC,Comercio exterior, 2011). Dans l'ensemble, les perspectives pour 2011 sont excellentes avec une hausse de 17\% par rapport à 2010, les exportations totales devraient atteindre 81 milliards de dollars, soit le double du montant en valeur atteint en 2005 et le triple de celui de 2001. Les importations devraient croitre fortement, probablement de $28 \%$ en 2011, en raison de la croissance soutenue et de l'appréciation du peso. L'ampleur des excédents de la balance commerciale devrait être réduite mais rester à un niveau confortable (2\% du PIB, voir Pagina 12 du 3 janvier 2011).

24 Voir Comercio Argentina- Brasil, una perspectiva desagregada (2009, 1-8), note technique $\mathrm{n}^{\circ} 15$, Ministerio de economia y finanzas publicas. En 2010, selon l'INDEC, la structure des exportations de l'Argentine vers le Brésil est la suivante: $33 \%$ de produits alimentaires, $32 \%$ de produits industriels, $25 \%$ de produits primaires et $10 \%$ de combustibles. $42 \%$ des exportations industrielles de l'Argentine sont destinées au Brésil. Le poids de l'industrie automobile est très important : $81 \%$ des exportations d'automobiles et $65 \%$ des pièces détachées sont vendues au Brésil. La structure des importations de l'Argentine provenant du Brésil est: 19\% de machines, $30 \%$ d'équipements, $19 \%$ de pièces détachées, $10 \%$ d'automobiles, $12 \%$ d'articles de consommation et $11 \%$ de combustibles Selon Gigliani G. et Perrone G, les exportations industrielles totales de l'Argentine rassemblées dans la rubrique produits manufacturés d'origine industrielle (MOI) ont triplé entre 2002 et 2008, et celles destinées au Brésil ont augmenté légèrement plus (3,5 fois), mais les importations provenant de ce pays ont été multipliées par 7,5. Gigliani G. et Perrone G (2009): "Argentina, peso devaluado y agravamento del deficit industrial, 2002-2008" in III Jornadas de economia politica (p. 4,1-16). L'essentiel de ce déficit avec le Brésil s'explique par les achats de machines, appareil et matériels électriques. 
augmente - y compris dans le secteur industriel - mais à un rythme aujourd'hui moins soutenu. La pauvreté baisse mais la forte inflation limite la progression du pouvoir d'achat des catégories les plus vulnérables. Les emplois informels, salariés ou non, perdent de leur importance relative, la balance commerciale est fortement excédentaire, le taux d'endettement n'est pas excessivement élevé et on peut s'attendre à ce que dans un avenir proche l'Argentine renoue avec les marchés financiers internationaux, enfin les réserves internationales augmentent malgré les sorties massives de capitaux en 2008 et 2009.

L'appréciation récente du taux de change réel, la quasi-inexistence d'une politique industrielle ciblée, et enfin une structure du système fiscal qui reste largement régressive ${ }^{25}$ sont de nature à fragiliser le modèle argentin. Cependant, sauf un choc externe provoqué par une reprise de la crise internationale et la chute du cours des matières premières, à court et moyen terme le cercle économique vertueux dont bénéficie l'Argentine devrait perdurer tout en perdant de son dynamisme.

Une des causes de la réindustrialisation de l'Argentine réside dans la politique de change choisie. Celle-ci s'apparente de facto à une politique de changes multiples. Malgré l'appréciation récente du taux de change, ce dernier reste relativement déprécié. Un tel taux de change protège l'industrie, favorise une nouvelle substitution des importations et permet une hausse des salaires réels sans que celle-ci ait nécessairement un impact important sur les salaires exprimés en dollars. Le jeu combiné de la hausse des salaires et de l'emploi a des effets positifs sur la croissance tant que le solde de la balance commerciale reste positif. Les taxes (" rétentions") sur les cours des matières premières sont équivalentes à un taux de change apprécié pour les exportateurs de matières premières puisque chaque dollar obtenu est converti en moins de pesos. Ces taxes alimentent le budget et permettent de lever la contrainte budgétaire. La contrainte externe (solde de la balance commerciale) et la contrainte interne (excédents primaires au lieu de déficit), étant levées, la poursuite de la croissance rencontre moins d'obstacles et peut se réaliser à un rythme soutenu pour autant que ne réapparait pas une reprise de l'inflation.

Le principal obstacle à la poursuite de la croissance et à l'amélioration des indicateurs sociaux ne se trouve donc pas dans la contrainte externe comme

25 Nous ne pouvons ici traiter de cette question très importante. Pour l'Argentine, voir Gagerro J (2008) La progressividad tributaria: su origen, apogee y extravio, document de travail $n^{\circ} 23$ CEFID*AR, Gomez Sabaini et Rossignoo D. (2008): Argentina, analisis de la situacion tributaria y propuestas de reformas impositivas destinadas a mejorar la distribucion del ingresso, Working Paper, Universidad de Buenos Aires;Goni E., Humberto Lopez J. Et Serven L. (2008): Fiscal Redistribution and Income Inequality in Latin America, Policy Research Working Paper, n 4487. 
c'était le cas auparavant. Hier, la croissance entraînait des déficits croissants de la balance commerciale et conduisait à des dévaluations de la monnaie nationale, à une politique de contention de la demande, à une crise économique. Une politique de relance économique était alors décidée. Le retour de la croissance alimentait alors de nouveau un déficit de la balance commerciale. Cet aspect " pendulaire" des politiques économiques a pu être dépassé avec la hausse du cours des matières premières. Aujourd'hui la contrainte externe ne joue plus grâce à la hausse des cours internationaux du soja, sauf en cas de choc externe provoqué par une forte réduction de la demande des pays asiatiques. Bien au contraire le solde positif de la balance commerciale facilite la mise en œuvre de politiques expansionnistes. L'obstacle a la poursuite de la croissance n'est plus le même. Aujourd'hui, l'accélération de la hausse des prix met en péril le modèle argentin. Aussi est-il probable qu'aux lendemains des élections présidentielles la présidente réélue opte pour une politique de contention de la demande globale au lieu de chercher à développer l'offre en stimulant l'investissement dans le secteur industriel et dans les infrastructures (énergie, transport) afin de freiner la hausse des prix. Agir sur la demande globale peut réduire le taux d'inflation, mais au prix d'un ralentissement de l'activité économique et sans que les causes structurelles de l'inflation situées du côté de l'offre ne soient atténuées.

Si un tel choix devait être fait, il conduirait à un ralentissement de la hausse des salaires nominaux, à une réduction du taux de croissance du PIB et une création d'emplois plus faible. Le coût social de la réduction du taux d'inflation pourrait être conséquent. La base sociale du gouvernement pourrait être affectée par de telles mesures et entraîner un déficit de légitimité. Celui-ci rendrait plus difficile des résister aux lobbies du secteur agro-exportateur et de poursuivre avec la même ampleur la politique de taxation du cours international du soja. Le conflit distributif ravivé entre le secteur agro-exportateur et le gouvernement pourrait rendre plus difficile le financement des subventions visant à limiter l'impact des hausses des cours internationaux de l'énergie et des produits agricoles sur le marché intérieur. Le cercle vertueux, menacé par l'accélération de la hausse des prix pourrait alors devenir vicieux.

\section{MERCI}

Je remercie Jorge Marchini, Claudio Katz, Ruben Lo Vuolo, de nombreux amis argentins qui, par leurs discussions, m'ont aidé à mieux comprendre l'Argentine et bien sûr JM 